\title{
Travaux d'assainissement au Brésil
}

\section{Drainage work in Brazil}

\author{
PAR H. ALVARENGA \\ INGÉNEYR at DEPARTAMENTO NaClONal DE OHHAS DE SANEAMENTO (DNOS)
}

\begin{abstract}
Existence, au Brésil, de zones sujettes à tnondations périodiques. Création d'un organisme chargé d'organiser la lutte contre le fléau, avec gain simultané de terrains jadis submergés. Les méthodes de travail sont modernes et mettent en auvre un matériel puissant. La régulation du nivean dans les lagunes colières soumises à de ficheuses alternances de remplissage et de vidage est assurée par le maintien, en état d'ouverture, d'un émissaire pour les eaux lagunaires; descriplion du dispositif adople. Necessité de mettre les ouvrages d'art en harmonie avec le role acru qui leur esl dévolu. La protection des villes de Juiz de Fora et de Jorlo Alegre a mis en tante des solutions originales et audacieuses. Description de six noubeaux barrages hydro-électriques.
\end{abstract}

En 1935, le gouvernement du Brésil a fortement intensifié études et travaux pour assainir la partie litlorale de l'Etat de Rio de Janeiro, voisine de la capitale fédérale. Cette grande plaine, d'une largeur moyenne de $50 \mathrm{~km}$, esi connue sous le nom de Baixada Fluminense. Elle est au débouché d'un ensemble de bassins hydrographiques courrant environ $17.000 \mathrm{~km}$.

Dès le début, les travaux révélèrent leur intérêt de facon indisculable, ce qui incita le gouvernement à en envisager l'extension sur le plan national, grâce à un nouvel organisme, créé en 1940, l'actuel Departamento Nacional de Obras de Saneamento (DNOS). Le DNOS est chargé de l'asséchement et de la récupération, à des fins agricoles, des régions inondées ou inondables, ainsi que de la protection des villes contre les crues. Son activité se manifeste également dans le cadre de certains plans régionaux d'électrification, en se chargeant parfois d'une partie importante des travaux.

\section{Récupération des terres}

Le plus souvent, les travaux de récupération consistent à creuser un nouveau chenal pour les

\begin{abstract}
Existence in Brazil of areas subject to periodn flooding. Creation of an authority charged with organising the fight against plague, with the simultaneous reclamation of previonsly submerged land. The working methods are modern and make use of powerful equipment. Regntation of the level in corstal lagoons, subject to disturbing alternations of filling and emptying, is ensured by kceping an emissary open for the lagoons ibaters; description of the method adopted. Necessily of adupting structures to their increased activities. Protection of the towns of Juiz de Fora and Porto Alegre has resulted in bold and original solutions. De'scription of six new hylro-electric arms.
\end{abstract}

rivières qui nourrissent les zones maréagenses et à endiguer les basses vallées (maritime et fluvio-maritime) dans lesquelles le niveau des grandes crues est fréquemment au-dessus du terrain naturel.

Les digues sont généralement construites en terre, en utilisant les deblais du canal principal et des canaux secondaires.

La section transversale des digues en terre est trapézoïdale, avec 3 à $6 \mathrm{~m}$, ou davantage, de largeur en crête: la pente des lalus atteint $1 / 2$ du còté de la rivière el $1 / 4$ à $1 / 6$ du cóté de la terre. Leur hateur moyenne, sur le littoral brésilien, est de $3 \mathrm{~m}$. Dans certains cas spéciaux, elles onl cité portés à $6 \mathrm{~m}$.

Les petits «polders » ainsi formés sont convenablement drainés vers les collecteurs, qui communiquent avec la rivière par l'intermediaire de vannes automatiques ou de stalions de pompage.

Naturellement, des pompes ne sont installées que dans les « polders » dont l'exploitation interdit toute inondation el où le rendement économique justifie un tel investissement.

Plus haut dans ces vallées, les crues peuvent, en général, être contenues dans le lit lui-même, s'il est entretenu par dragage; le drainage est 


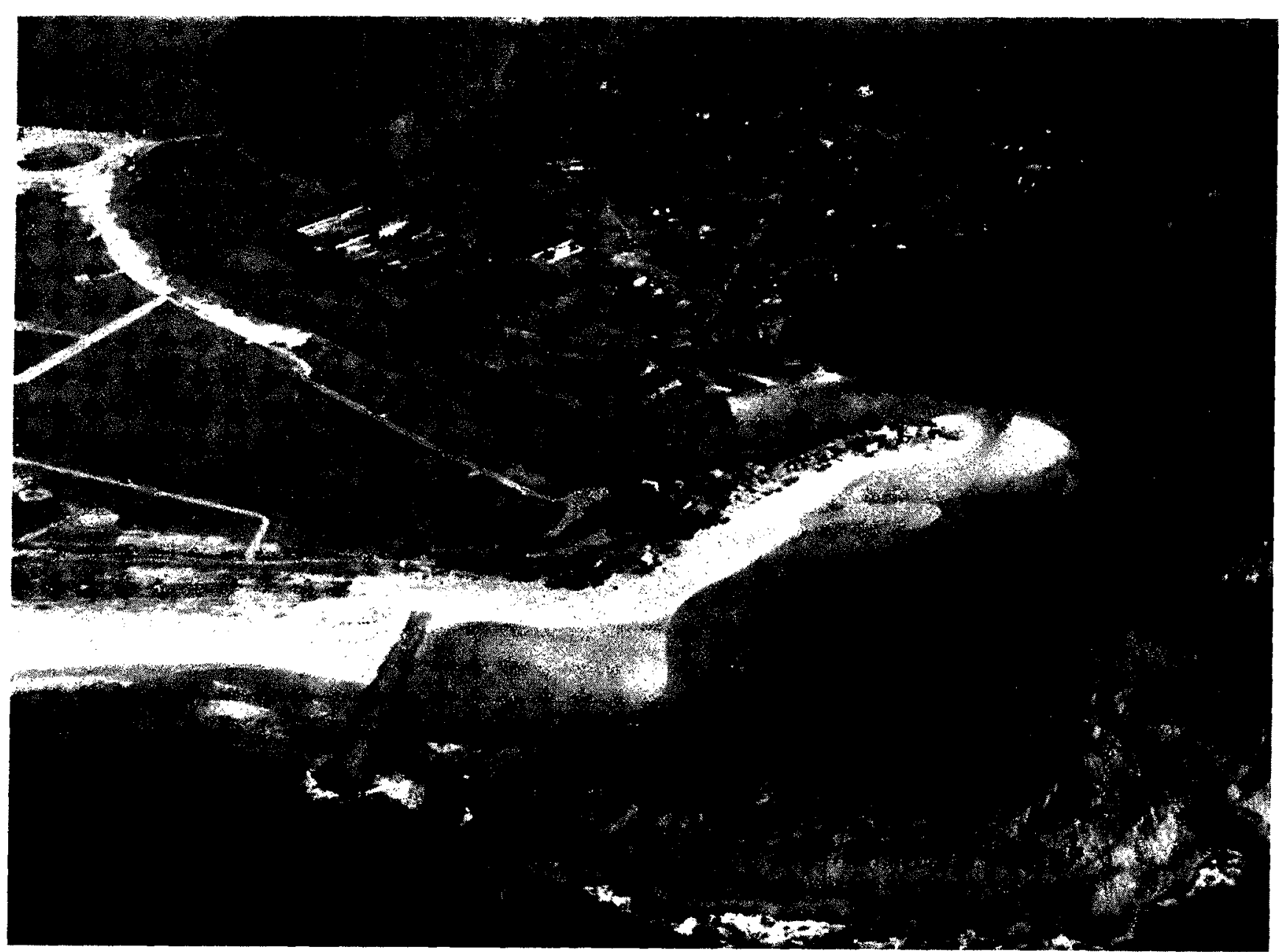

Fici. 1. - Lagune de Jacarepaguá.

Vue de l'epi de fixation de la bare du camal dembonchure.

Fia. 2. - Rio Guandu-Açu. Vue générale de la partie limilé par los digues en terre.

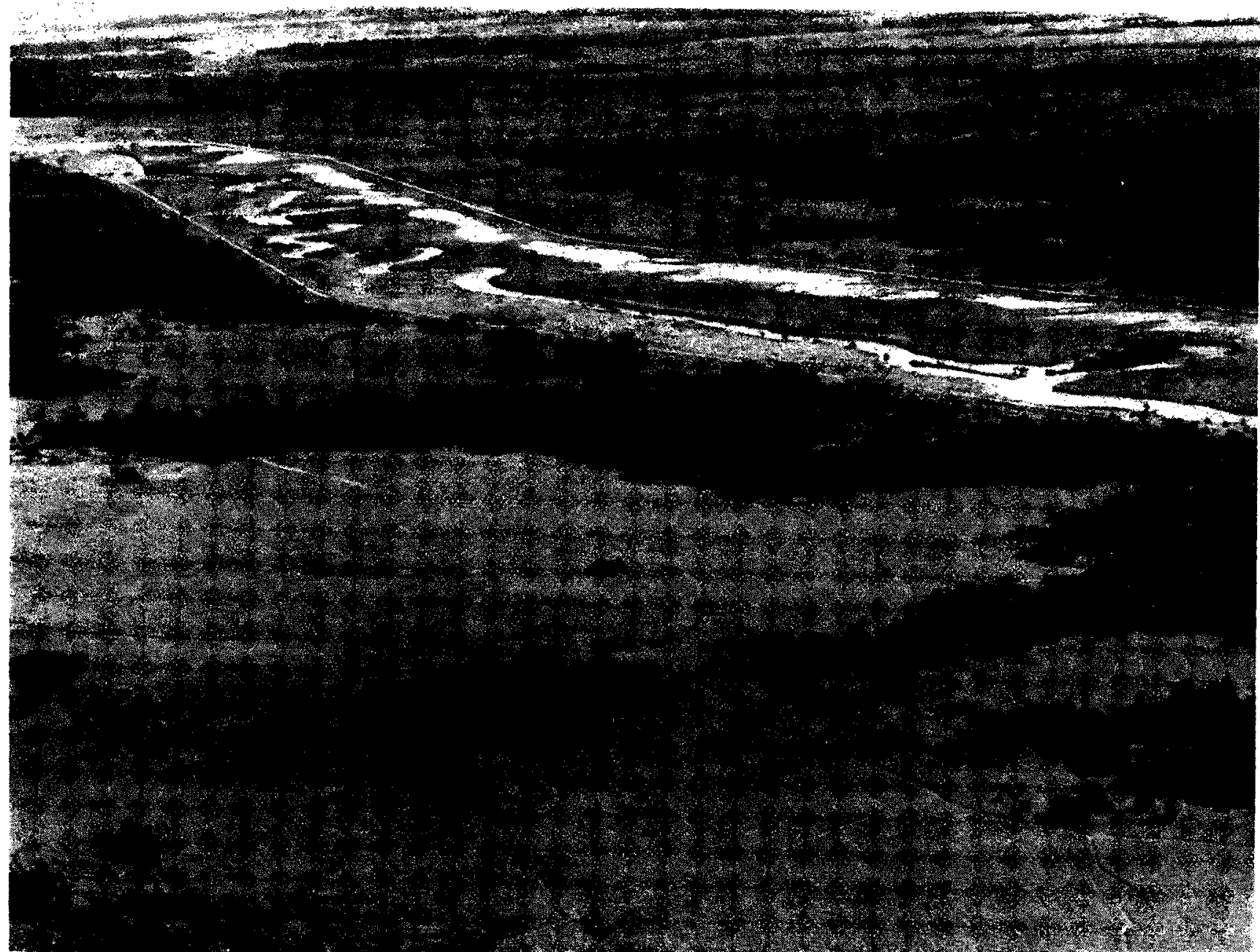




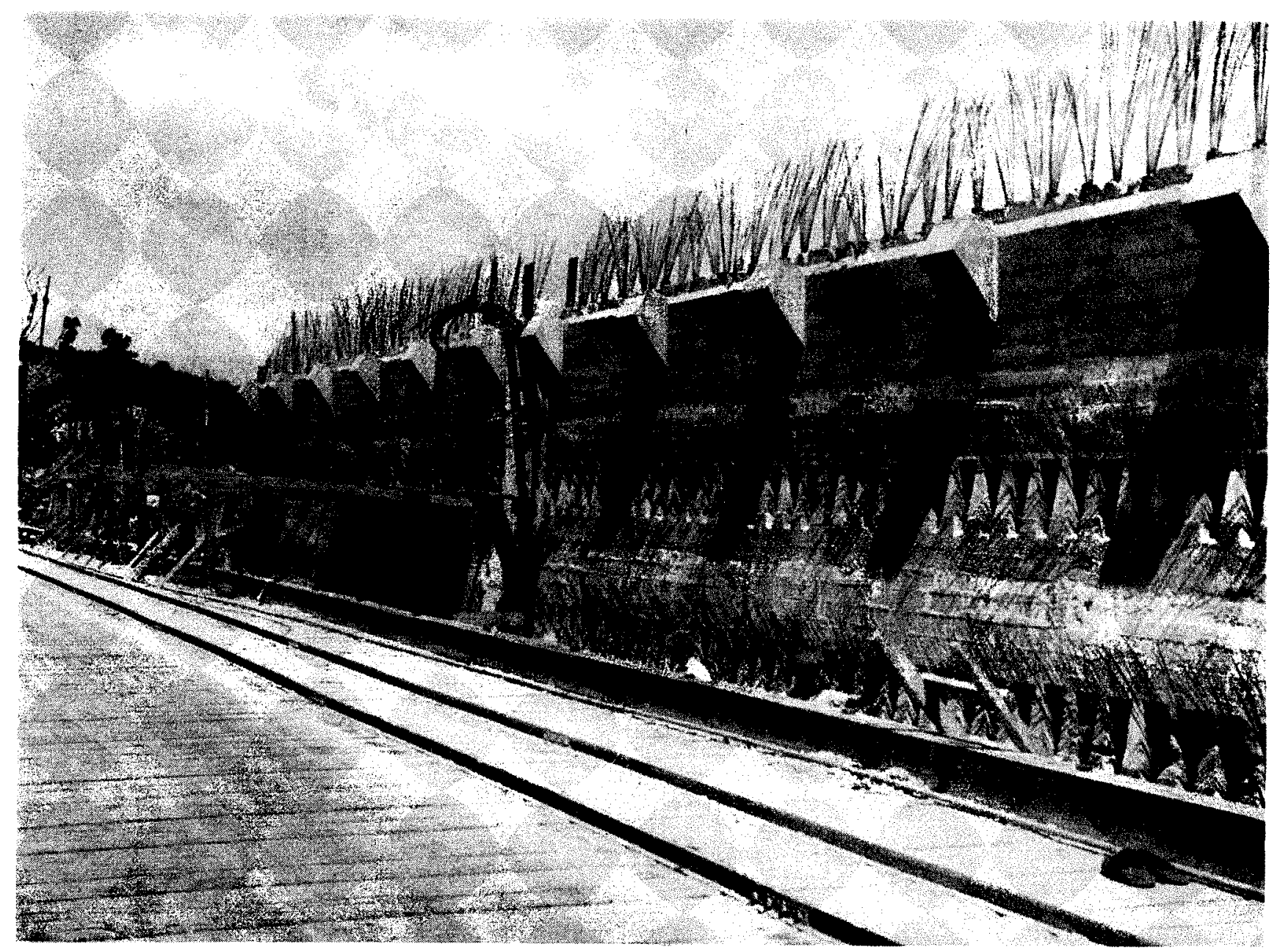

Fia. 3, - Barrage d'Ernestina, en beton precontmint.

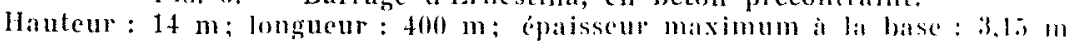

direct et ne pose pas de problemes trop complexes.

Lorsque les camaux projetés traversent des zones marécageuses étendues où des relevés topographiques seraient difficiles, on combine la photographie aćrienne et le nivellement lerrestre. Ce procédé simplific, tout en donnant une approximation suffisante pour le type de projet à élaborer, réduit grandement les travaux sur le terrain, particulièrement pénibles en zones marécageuses, et permet une íconomic appréciable de temps, dargent el de matériel.

Le tracé en plan du canal est exécuté sur la carte obtenue grâce aux photographies aériennes, puis, grâce aux cotes de quelques sections transversales assez exactement localisées, on ctablit son profil en long.

Les travaux effectués depuis 1936, soit dans divers marais du littoral, soit à l'intérieur, représentant le dragage de $2.900 \mathrm{~km}$ de canaux ou un volume total de déblais de $95.000 .000 \mathrm{~m}^{*}$.

Ces résultats n'ont pu être obtenus que par l'emploi massif de puissants draglines, dont plus de 180 unités, achetés par le DNOS, cons-

Fig, 4. - Barrage d'Ernestina. Détail des cables de précontrainte.

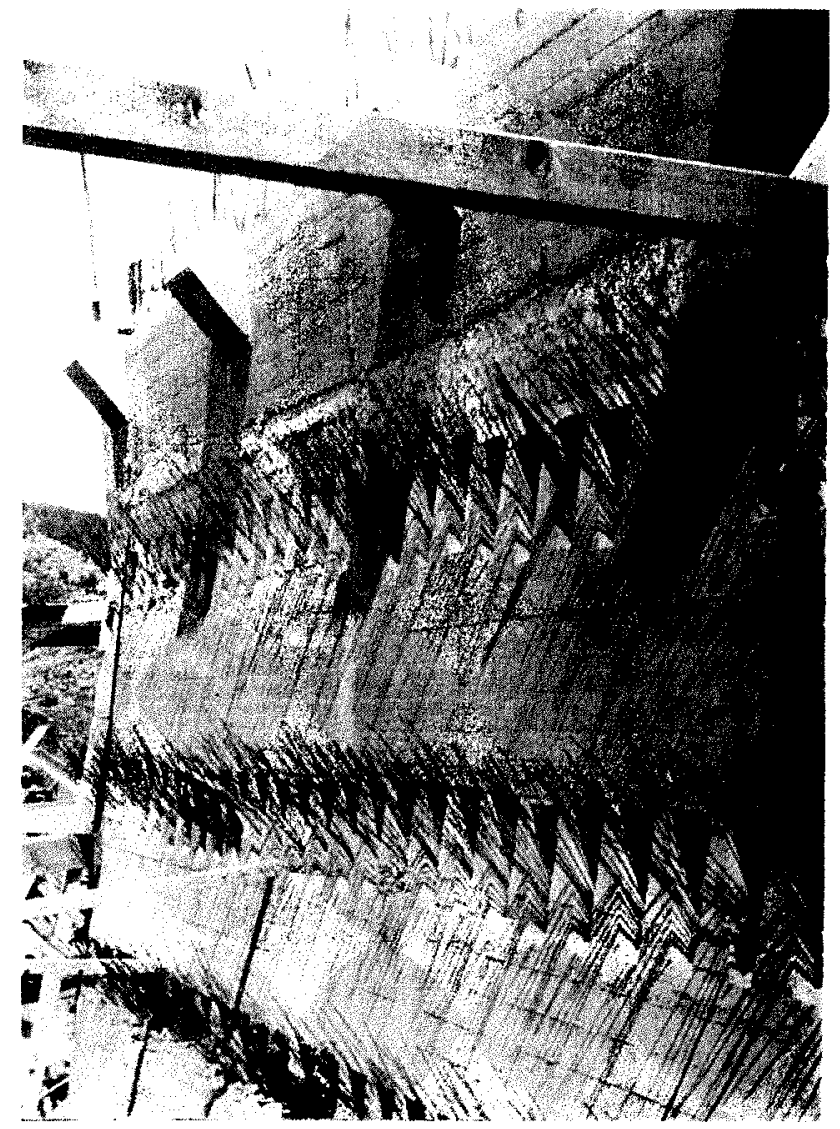


tituent la plus grande flotle du monde. Grâce à leur mobilité, à leur facilité de manouvre et à leur prix relativement peu élevé, ces engins ont toujours été utilisés avec succès, même dans les pires terrains.

En les installant sur d'épaisses plates-formes cn bois, afin de diminuer la charge du terrain, tous les marécages ont pu ètre franchis par des machines pesant jusqu'à 60 tonnes.

L’usage génćralisé de cet appareillage, sur n'importe quel terrain, a simplifié les travaux, car l'emploi de dragues flottantes est limitẻ aux canaux dont la profondeur d'eau est suffisante; les ponts et les rapides rendent, d'autre part, leurs déplacements difficiles.

Les « draglines » ont permis d'atteindre les extrémités des divers bassins, offrant ainsi un domaine d'application beaucoup plus étendu.

\section{Liaison permanente entre les lagunes côtières et la mer}

Il est relativement fréquent de rencontrer, sur le littoral brésilien, des rivières débouchant dans les lagunes côtières fermées par des barres instables.

Quand l'eau monte dans ces lagunes, le cordon littoral se rompt, provoquant un violent courant de chasse qui fait rapidement baisser le niveau. Les lagunes une fois vidées partiellement, l'action de la mer redevient prédominante, la barre se reforme, et la brèche ouverte dans le cordon littoral est bouchée.

Pendant les périodes de hautes eaux dans la lagune, le niveau des affluents demeure élevé et des débordements se produisent dans les basses vallées.

Du point de vue hydraulique, la solution consiste à maintenir la communication toujours ouverte. Quand la barre est appuyée sur l'une des rives, à une saillie rocheuse naturelle, on peut y parvenir en construisant, a partir de la rive sablonneuse, une jetée s'avançant vers le large au-delà de la ligne neutre, de manière à dévier les sables charriés.

Les jetées sont construites en blocs de carrière disposés de façon à former une section transversale trapézoïdale arec talus à faible pente. L'extrémité de la jetée atteint la zone où les courants sont suffisamment rapides pour empêcher les dépòts. Dans cette partie, son orientation est parallèle à la direction de l'entraînement. Ainsi, tout déplacement de sables le long de la plage est intercepté par la jetée. Les particules qui atteignent l'extrémité sont déjà orientées dans le sens des courants qui les entrainent. La plage s'engraisse évidemment sur la face externe de la jetée, dans la zone où la vitesse des courants permet aux sables de se déposer. C'est ainsi qu'à Jacarepaguá, la construction de la jetée fail progresser la plage d'environ $50 \mathrm{~m}$. Au-delà de cette limite, les sables sont entraìnés. Il y a huit ans que la passe se maintient toujours ouverte et le niveau maximum dans la lagune a diminué de $1 \mathrm{~m}$.

Quand le cours d'eau débouche sur une plage de sable (cas moins fréquent), on a recours à la construction de deux jetées convenablement orientées par rapport à la direction du cheminement littoral.

Ces méthodes ont déjà permis de fixer deux barres dans la Baixada Fluminense : celle de Jacarepaguá, dans le District Fédéral, et celle de Ponta Negra, à Maricá, dans l'Etat de Rio de Janeiro.

\section{Travaux complémentaires}

Le dragage des canaux a amené le DNOS ì exécuter de nouveaux ouvrages d'art en remplacement des anciens, dont la portée était insuffisante. Sur les voies ferrées et sur les routes, ont été construits de nouveaux ponts; d'autres on pu être conservés après augmentation de leur portée et reprise en sous-œuvre de leurs fondations. On a construit des viaducs sur les traversées des digues. Sur les petits affluents, un grand nombre de fossés de drainage a été creusé ou aménagé par élargissement et approfondissement.

Le nombre de ponts réalisés s'élève aujourd'hui à 553, parmi lesquels 153 en béton armé.

\section{Défense des villes contre les inondations}

Quelques villes brésiliennes, périodiquement inondées par les rivières qui les traversent, ont demandé au DNOS d'organiser leur protection. Juiz de Fora, la ville la plus industrielle de l'Etat de Minas Gerais, inondée par les crues de la rivière Paraibuna; Porto Alegre, constamment menacé par le Guaiba, et d'autres cités de moindre importance.

La solution adoptée pour Juiz de Fora a consisté à ouvrir à la rivière un nouveau lit sur $21 \mathrm{~km}$, en tenant compte de l'importance actuelle de la ville et de son développement ultérieur. Tous les ponts ont été réaménagés, le nouveau lit, de section trapézoïdale, ayant $33 \mathrm{~m}$ de largeur au plafond et des talus à 1/1. Dans certains tronçons, les berges ont été revêtues en maçonnerie. A l'amont, un réservoir d'accumulation a été prévu pour régulariser les débits entre un maximum de $340 \mathrm{~m}^{3} / \mathrm{s}$ et un minimum de $10 \mathrm{~m}^{3} / \mathrm{s}$ correspondant aux besoins de quatre usines hydro-électriques situées à l'aval.

Le problème de Porto Alegre est plus vaste. 


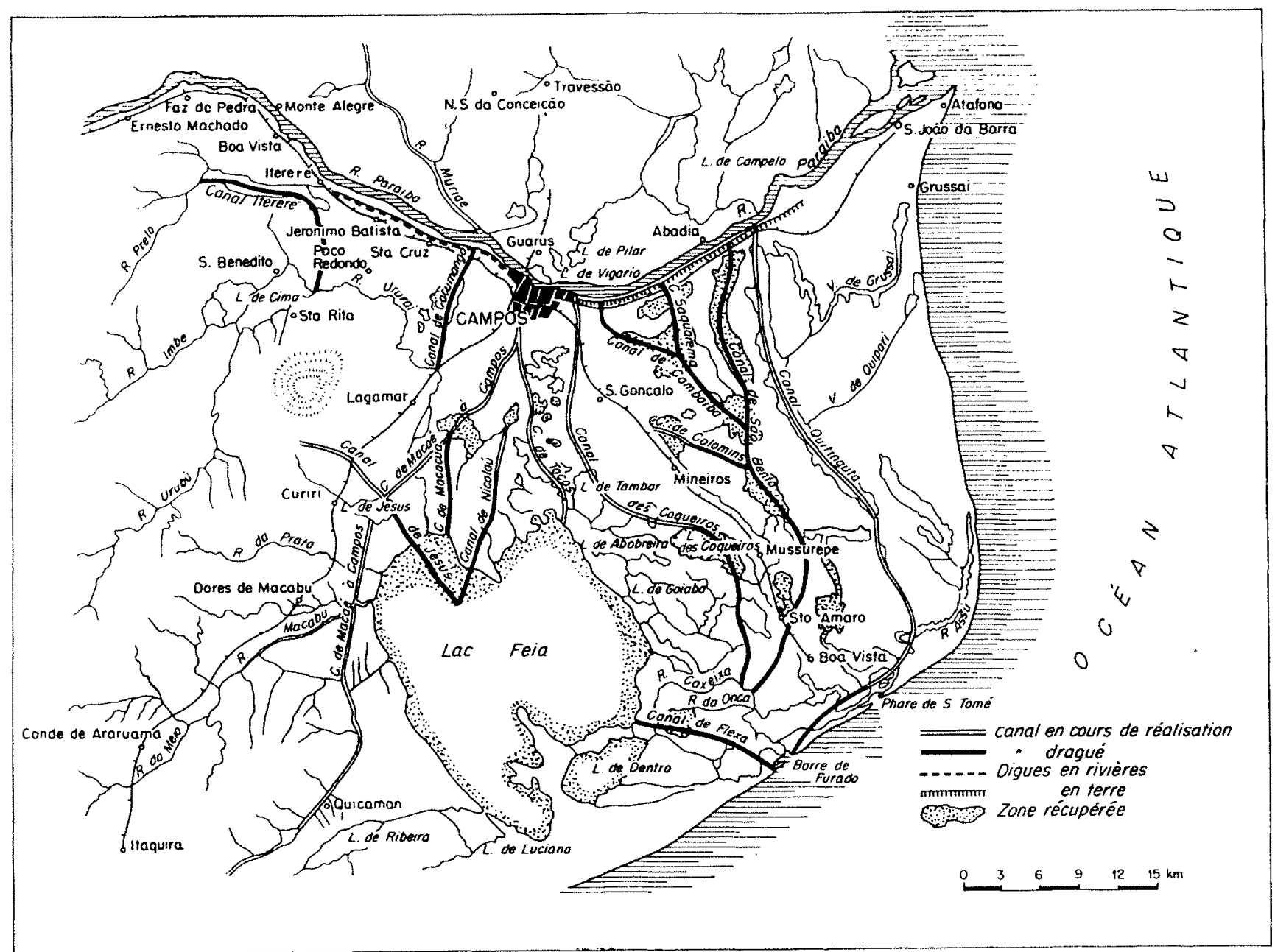

Fis. 7. - Schema des lravax de defense contre les inondations of de dramage de la rive droite du Rio Parabia.

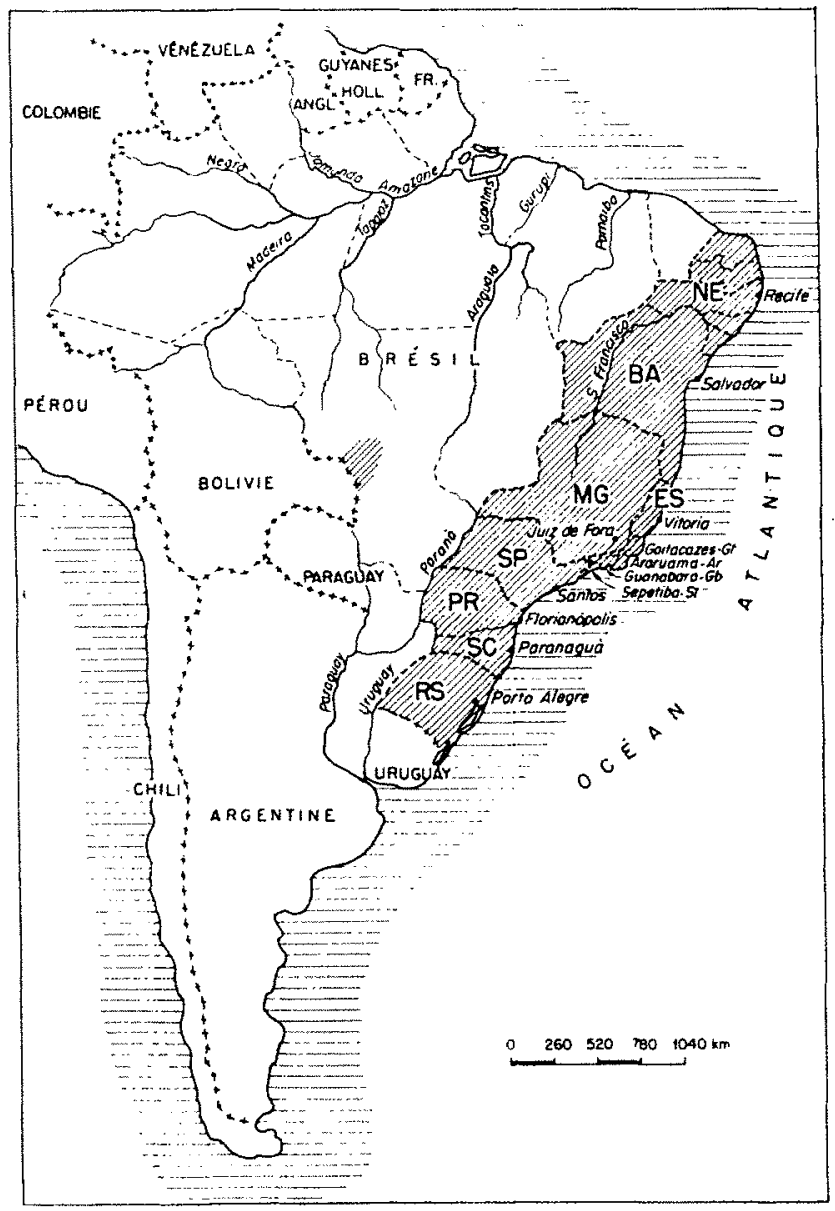

Les grandes crues de la rivière Guaiba, qui alteignent un débit maximum de $35.000 \mathrm{~m}^{3}$ par seconde, inondaient la zone portuaire, commerciale et industrielle de la ville. La grande crue de 1941 est montéc jusqu’à $1,63 \mathrm{~m}$ au-dessus des quais.

La solution qui s'imposait était l'endiguement des rives. Le projet de Porto Alegre comporte, en particulier, la réalisation d'une digue contournant la ville. Son tracé et ses caractéristiques sont naturellement conditionnés par les données locales, telles que l'exploitation du porl ou l'urbanisme.

Une première tranche comporte une digue en terre contournant la plaine jusqu'aux quais du port; elle a $8 \mathrm{~m}$ de largeur en crête et des talus à $1 / 2$.

Pour le port, en raison des difficultés de réalisation d'une digue dans la ville, il a fallu empiéter sur l'estuaire. Un quai en béton armé, de $5.000 \mathrm{~m}$ de longueur, a été construit; il constitue un mur de soulènement pour un terre-plein s'étendant sur $1 \mathrm{~km}^{2}$ et représentant 5 millions de $\mathrm{m}^{3}$ de remblai. Le quai est presque achevé $\mathrm{cl}$ le remblayage hydraulique très avancé.

Fig. 8. - Zones de travail du departement national des travaux d'assainissement (DNOS). 
La surfice défendue est de $18 \mathrm{~km}^{2}$. Pendant les crues, les précipilalions locales seront evacuées par un systeme de canaux el de pompes d'une capacite tolale de $18 \mathrm{~m}^{3} / \mathrm{s}$ pour une hauteur de refoulement de $6 \mathrm{~m}$. Les hauts quartiers de la ville seront drainés par un canal circulaire débouchant directement dans la rivière par gravité.

\section{Barrages}

En collaboration avec l'Elat de Rio Grande do Sul, le DNOS réalise tout un ensemble de barrages prévus dans le plan général d'électrification de l'Etat. Il est également chargé de la construction des galeries d'amence.

Les barrages de Salto, de Capingui et d'Ernestina, qui font partie du plan général, sont terminés.

Le barrage de Salto, situé sur la rivière Santa Cruz, accumule 13 millions de $m^{3}$ d'eau, qui sont dérivés grâce à un tunnel de $2.087 \mathrm{~m}$ vers la vallée de la riviere Santa Maria. La chute utilisée est de $680 \mathrm{~m}$ et la puissance totale s'clève à $85.000 \mathrm{ch}$. Le barrage a $15 \mathrm{~m}$ de hauteur et $580 \mathrm{~m}$ de longueur. C'est un barrage poids, en béton cyclopéen, à profil triangulaire; son tracé en plan est circulaire. I.e tunnel d'amenée a $2.087 \mathrm{~m}$ de longueur, 2 m de diamelre el une capaciti de $7,320 \mathrm{~m}^{*} / \mathrm{s}$.

Le barrage de Capingui est également en béton cyclopéen. Il a $23 \mathrm{~m}$ de hauteur, $220 \mathrm{~m}$ de longueur el un rayon de courbure de $150 \mathrm{nt}$. II retiendra 40 millions de $\mathrm{m}^{3}$.

Le barrage d'Ernestina est en héton pré-contraint. Il a $400 \mathrm{~m}$ de lonsueur, $14 \mathrm{~m}$ de hauteur et seulement $3,15 \mathrm{~m}$ de largeur à la base. Encastré dans le rocher, il travaille a la flexion.

Trois autres barrages sont en cours de réalisation : José Maia Filho, Blang et Canastra.

Le barrage José Maia Filho est en béton cyclopéen. Il a $358,50 \mathrm{~m}$ de longueur et $23 \mathrm{~m}$ de hauteur. Son profil est triangulaire.

Le barage du Passo do Blang est en béton cyclopéen et possède un déversoir de $220 \mathrm{~m}$ de longueur, linité latéralement par des murs également en béton cyclopéen. Le développement total du barrage est de $720 \mathrm{~m}$ el sa hauleur maximum est de $20 \mathrm{~m}$.

Le barrage de Camastra est en béton armé, type Ambursen, à contreforts. Il a $179 \mathrm{~m}$ de longueur et $26 \mathrm{~m}$ de hauleur maxinum. Il est formé d'une dalle frontale d'étanchéité, inclinée à 45", constituant le parement amont et sappuyant sur 32 contreforts.

Toujours dans le cadre de sa collaboration avec les Etats, le DNOS construil un barrage en béton eyclopéen dans l'Etal de Sanla Catarina pour la production d'énergie checlrique, of deux barrages en terre pour la défense contre les crues de la ville de Bela Horizonle, capitale du Minas Gerais.

Le programme prévoit, pour 1955, la construction d'un grand barrage en béton cyclopéen, Ie harrage de Suissa, dans l'Etat de EspiritoSanto, barrage qui pernettra l'installation d'une puissance de $70.000 \mathrm{ch}$. Ce barrage doil avoir $46 \mathrm{~m}$ de hauteur et $270 \mathrm{~m}$ de longueur.

\section{Conclusion}

En dix-huil ans d'aclivité, le DNOS a réalisé les travaux suivants, pour ne ciler que les principaux :

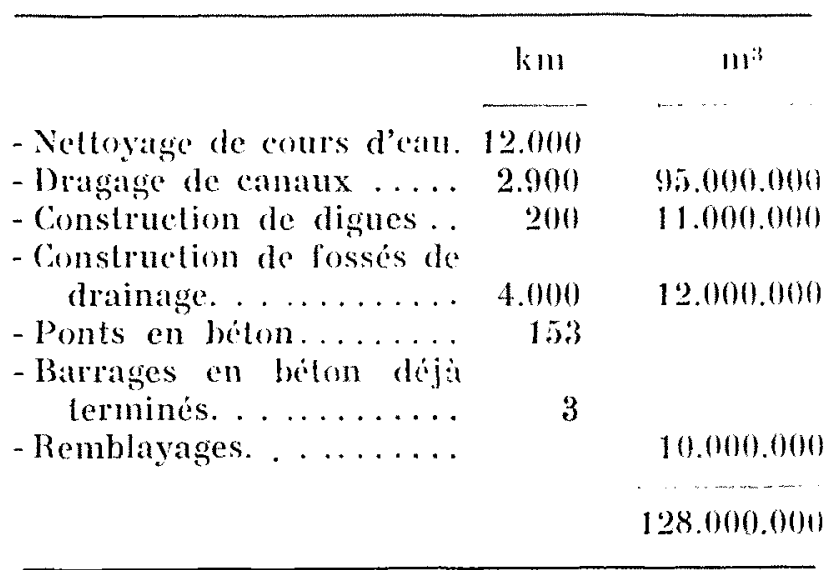

Ces chifres illustrent parfaitement, dans leur secheresse, l'ampleur de l'effort accompli et des moyens mis en couvre. 